\title{
Pancreatic Fat Infiltration Is Associated with a Higher Risk of Pancreatic Ductal Adenocarcinoma
}

\author{
Vishal Desai $^{\mathrm{a}} \quad$ Kevin Patel $^{\mathrm{b}} \quad$ Ravi Sheth $^{\mathrm{c}} \quad$ Usman Barlass $^{\mathrm{a}} \quad$ Yuet-Ming Chan $^{\mathrm{a}}$ \\ Joy Sclamberg ${ }^{c}$ Faraz Bishehsaria $^{\mathrm{a}}$ \\ aDepartment of Internal Medicine, Division of Gastroenterology, Rush University Medical Center, Chicago, IL, USA; \\ b University of Wisconsin School of Medicine and Public Health, Madison, WI, USA; 'Department of Radiology, Rush \\ University Medical Center, Chicago, IL, USA
}

\author{
Keywords \\ Pancreatic cancer - Fatty pancreas - Alcohol · Metabolic \\ syndrome
}

\begin{abstract}
Background: Pancreatic ductal adenocarcinoma (PDAC) has a poor survival rate, partly due to delayed diagnosis. Identifying high-risk individuals could lead to early detection and improve survival. A number of risk factors such as alcohol consumption and metabolic syndrome are associated with fatty infiltration of the pancreas. Experimental models show that a fatty pancreas promotes tissue inflammation and fibrosis, which could promote PDAC. Methods: We conducted a case-control study in a single-university tertiary hospital. Sixty-eight PDAC cases with recent non-contrast computed tomography (CT) and 235 controls were studied. The controls had no history of malignancy and underwent CT colonography for cancer screening in the same period. Pancreatic fat was estimated by calculating pancreatic $(P)$ attenuation, corrected to splenic $(S)$ attenuation, measured in three $1.0-\mathrm{cm}^{2}$ regions of the pancreas. The P.S100 value calculated was used to estimate fatty infiltration of the pancreas (FIP), with a lower P.S100 representing a higher FIP. Results: The PDAC patients had a lower BMI and a higher rate of type 2 diabetes mellitus. The P.S100 was lower in cases than in con-
\end{abstract}

trols (86.452 vs. 92.414, $p=4.016 \mathrm{e}-06)$, suggesting that FIP is higher with PDAC. The risk of developing PDAC steadily increased significantly for the quartiles with a higher FIP compared to the low FIP quartile. No correlation between BMI and FIP ( $r=-0.1031179 ; 95 \%$ confidence interval [Cl] -0.22267106 to 0.01949092 ) was found. Adjusting for confounders (age, sex, BMI, and DM), the risk of developing PDAC according to the FIP was estimated to be $3.75(95 \% \mathrm{Cl}$ 1.9234408-7.993337; $p=0.000171)$. FIP was stable before and after the diagnosis of PDAC in 9 cases with prior CT scans when no pancreatic tumor was identifiable. Conclusion: Fatty pancreas is associated with an increased risk of pancreatic cancer. Once confirmed in larger-scale studies, these findings could help to identify at-risk individuals, particularly in high-risk groups such as chronic alcohol consumers

() 2020 S. Karger AG, Basel

\section{Introduction}

Pancreatic ductal adenocarcinoma (PDAC) is currently the fourth leading cause of cancer-related death with a 5 -year survival rate of 5-9\%. This dismal prognosis combined with the expected rise in the disease rate will place PDAC as the second-most common cause of cancer-related death in the USA by 2030 [1-4]. The poor survival rate 
is partly due to PDAC being a silent disease, with a lack of symptoms until the later stages of the disease; $52 \%$ of PDAC patients have distant disease and $29 \%$ have regional spread at the time of diagnosis $[4,5]$. Only a small portion of cases are candidates for surgical resection $[6,7]$ and, even after complete resection with negative margins, PDAC exhibits high recurrence rates and distant metastases [8-10]. Therefore, identifying at-risk individuals would help early detection, potentially leading to more effective treatments at the time of diagnosis and improving survival.

Features of metabolic syndrome (MetS) such as obesity, elevated BMI, and type 2 diabetes mellitus (DM) have been found to be associated with an increased PDAC risk and, more recently, such factors have been implicated in the ongoing increase in the disease rates [11-13]. Several lines of evidence suggest that features of MetS are associated with changes in the inflammatory milieu in the pancreas which result in tissue remodeling and fibrosis, promoting pancreatic carcinogenesis [14-16]. Fatty pancreas, an analog of fatty liver disease, is an understudied entity based on the deposition of fat in the pancreas. Fatty pancreas has been associated with chronic alcohol consumption as well as features of MetS, such as obesity and $\mathrm{DM}$, both in animal and human studies $[17,18]$. Fatty infiltration increases the expression of proinflammatory cytokines and extracellular matrix remodeling, leading to inflammation and fibrosis [13, 19-21]. As PDAC progression has been linked to increased levels of adipocytokines and tissue inflammation, it seems plausible that fatty pancreas may be linked to pancreatic cancer [22-24]. In fact, animal models of chemically induced and genetic models of PDAC found that fatty pancreas indeed predisposes to the development of pancreatic cancer [25-27]. Based on histopathological analysis, fatty pancreas was also found to be associated with PDAC in men [28].

While the gold standard for diagnosing fatty pancreas relies on pathology assessment of the whole tissue, this method is of limited applicability in living individuals and in clinical practice. Noninvasive imaging modalities can measure fat in tissues. In the past, imaging techniques were employed to estimate fatty pancreas based on computed tomography (CT) measurements of differences between pancreatic and splenic attenuation [11, 28-30]. Based on histopathological analysis, fatty pancreas was found to be associated with PDAC [28]. A follow-up study verified that CT attenuation index was correlated with histopathology-based assessment of the pancreas [20]. These studies established CT as a noninvasive method for estimating fatty infiltration of the pancreas (FIP).

In this cross-sectional study, we aimed to determine if fatty pancreas, determined by radiographic evaluation, is associated with a risk of developing PDAC. Based on the experimental evidence, we hypothesized that fatty pancreas is associated with an increased risk of pancreatic cancer.

\section{Methods}

\section{Patients}

We performed a retrospective review of patients diagnosed with pancreatic cancer at the Rush University Medical Center (RUMC) in 2006-2016. Sixty-eight consecutive cases of histologically proven PDAC with available non-contrast abdominal CT imaging prior to diagnosis were selected. Controls were 235 subjects with no history of malignancy in the past 10 years, who underwent CT colonography (also a non-contrast study), for colon cancer screening in the same period. Exclusion criteria included individuals with a known genetic syndrome associated with an increased risk of pancreatic cancer as well as those with known pancreatobiliary pathology (e.g., a history of acute/chronic pancreatitis and obstructive biliary stones).

Data were collected on demographics and relevant laboratory results (e.g., age, gender, BMI, and DM). Smoking and alcohol histories were available for all cases (191 [75.4\%]) and most of the controls (192 [75.8\%]). For study purposes, the diagnosis of DM and hyperlipidemia (HLP) were collected from the charts based on the ICD codes. Patient data were transferred to a nonidentifiable format and maintained on a secure Microsoft Excel and SPSS spreadsheet.

\section{Abdominal CT Measurements}

We followed the radiological method of quantifying pancreatic fat used by Kim et al. [31], which was corroborated by comparing $\mathrm{CT}$ and histological measurements of pancreatic fat. This consisted of internal correction of pancreatic attenuation $(\mathrm{P})$ to the individual splenic attenuation (S) on CT imaging without the use of intravenous contrast. On CT, areas of FIP appear hypoattenuated relative to normal tissue.

The area $\left(1.0 \mathrm{~cm}^{2}\right)$ of pancreatic attenuation was measured in 3 regions of interest (ROI) in the pancreatic head, body, and tail using a dedicated 3-dimensional computer workstation. These areas of attenuation were selected away from the site of the tumor to exclude the possibility of any reactive tissue remodeling adjacent to the tumor. The mean CT attenuation of the 3 ROIs was used as the representative measurement of the pancreas. The spleen was similarly measured by averaging 3 separate $1.0 \mathrm{~cm}^{2}$ ROIs. Pancreatic and splenic lesions, calcifications, vascular structures, and peritumoral tissue around the pancreas were excluded from these measurements.

We calculated the difference between pancreatic and splenic attenuation, i.e., $\mathrm{P}$ minus $\mathrm{S}$. To ensure positive values, we added 100 to each pure P minus S value, labeled as P.S100. The P.S100 value was used as an estimate of the fatty infiltration of the pancreas (FIP), with lower P.S100 translating to a higher relative FIP and a fattier pancreas.

\section{Statistical Analysis}

SPSS v23 (SPSS, Inc., Chicago, IL, USA) and $R$ v3.5.1 were used for all analyses. Numeric results are presented in the text as mean \pm SEM, unless otherwise specified, and were compared using parametric (Student's $t$ test) or nonparametric (Wilcoxon's signed-rank test) tests as appropriate. The $\chi^{2}$ test was performed to analyze the correlation between parameters. Logistic regression was conducted to calculate the odds ratio (OR) of having PDAC (the outcome variable), based on the pancreatic fat (the exposure variable) and adjusted for possible confounders (age, BMI, and DM). The adjusted ORs are presented with their $95 \%$ confidence intervals (CIs). The ORs of PDAC in the 1st, 2 nd, and 3rd quartiles of FIP values were compared with the rate in the lowest FIP (4th) quartile. 
Table 1. Baseline characteristics of the study participants

\begin{tabular}{lccc}
\hline & $\begin{array}{c}\text { Controls } \\
(n=235)\end{array}$ & $\begin{array}{l}\text { Cases } \\
(n=68)\end{array}$ & $p$ value \\
\hline $\begin{array}{l}\text { Age, years } \\
\text { Sex }\end{array}$ & $66.2 \pm 12.4$ & $68.4 \pm 10.5$ & 0.18 \\
$\quad$ Male & $67(28.5)$ & $32(47.0)$ & $<0.01$ \\
$\quad$ Female & $168(71.5)$ & $36(53.0)$ & \\
BMI & $27.3 \pm 5.4$ & $24.4 \pm 5.5$ & 0.0002 \\
Smoking & & & 0.8542 \\
$\quad$ History & $98(51.3)$ & $34(50.0)$ & \\
$\quad$ Never & $93(48.7)$ & $34(50.0)$ & \\
Alcohol abuse & $13(6.8)$ & $5(7.4)$ & 0.8712 \\
$\quad$ History & $178(93.2)$ & $63(92.6)$ & \\
$\quad$ Never & $128(67.0)$ & $44(64.7)$ & 0.76903 \\
Hypertension & $64(33.0)$ & $24(35.3)$ & \\
$\quad$ Yes & & & \\
$\quad$ No & $45(23.6)$ & $29(42.6)$ & 0.002772 \\
Diabetes & $146(76.4)$ & $39(57.3)$ & \\
$\quad$ Yes & & & \\
$\quad$ No & $88(47.1)$ & $25(36.8)$ & 0.1434 \\
Hyperlipidemia & $\quad 99(52.9)$ & $43(63.2)$ & \\
$\quad$ Yes & Normal & & \\
\hline
\end{tabular}

Values are expressed as mean $\pm \mathrm{SD}$ or $n(\%)$.

Table 2. Pancreatic cancer risk ratio based on quartiles of fatty infiltration of the pancreas compared to the least fatty pancreas (quartile 4)

\begin{tabular}{llll}
\hline & PDAC risk ratio & CI & $p$ value \\
\hline Quartile 1 & 4.56 & $2.0-10.37$ & 0.000022 \\
Quartile 2 & 3.83 & $1.66-8.8$ & 0.00068 \\
Quartile 3 & 1.4 & & \\
Quartile 4 & 1 & & \\
\hline
\end{tabular}

There was a trend towards $p<0.001$ across quartiles. CI, confidence interval.

\section{Results}

Clinical characteristics of the study participants at the initial diagnosis of pancreatic cancer or CT colonography are shown in Table 1. In total, 68 cases of PDAC and 235 controls were included. As expected, the PDAC cases had a lower BMI than controls $(24.4 \pm 5.5$ vs. $27.3 \pm 5.4$; $p<$ 0.01 ), and higher DM rates (42.6 vs. $23.6 \%$; $p<0.01$ ). There were more females in the control group (168/253 [71.5\%] vs. 36/68 [53.0\%] cases; $p<0.01)$. There was no significant difference regarding a history of smoking or alcohol abuse between cases and controls.

The P.S100 was significantly lower in cases than in controls (86.452 and 92.414, respectively; Wilcoxin test $=$ 5056; $p=4.016 \mathrm{e}-06$ ), suggesting a higher FIP among the

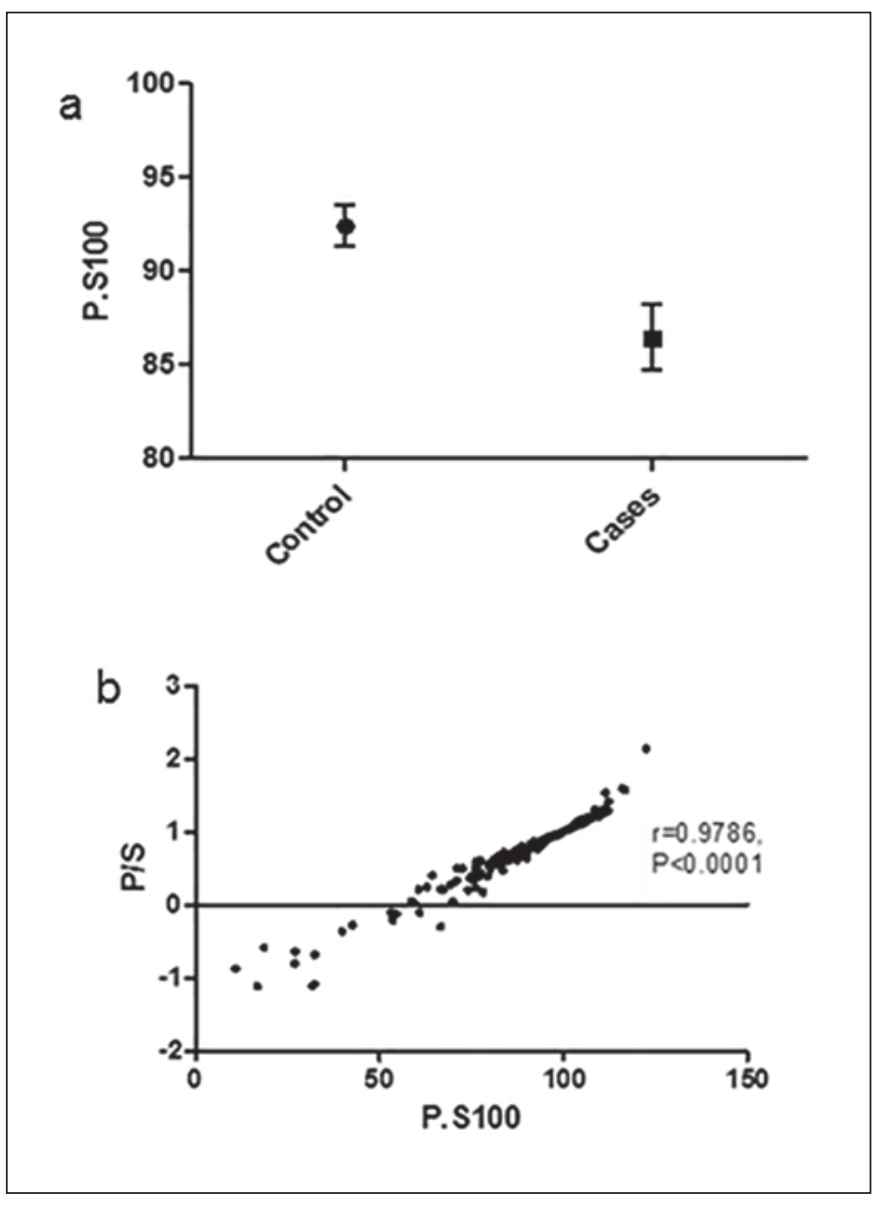

Fig. 1. a Fatty infiltration of the pancreas (FIP) values in 68 PDAC patients and 235 controls; mean \pm SEM. b Correlation between P.S100 and P/S ratio.

PDAC cases (Fig. 1a). The ratio of pancreas to spleen fat $(\mathrm{P} / \mathrm{S}$ ratio) revealed a similar result, with cases having a lower ratio than controls ( 0.6864 and 0.8249 , respectively; Wilcoxin test $=11,002 ; p=2.0 \mathrm{e}-06)$. There was a strong correlation between P.S100 and P/S ratio $(r=0.9786 ; p<$ 0.001) (Fig. 1b).

We then looked at the possible effect of BMI as a driving factor for the difference in FIP between cases and controls. Overall, the correlation between BMI and FIP was not significant $(r=-0.1031179 ; 95 \%$ CI -0.22267106 to 0.01949092). While the FIP was higher in PDAC cases, the BMI was higher among controls (Table 1), suggesting that the higher FIP in pancreatic cancer cases is independent of any possible BMI effect.

We then calculated the risk ratio of pancreatic cancer based on FIP (Table 2). For each increase in FIP quartile (i.e., a decrease in the P.S100 quartile) there was a significant increase in the risk of developing pancreatic cancer. With quartile 1 representing the fattiest pancreas, the pancreatic cancer risk ratio increased from the lowest to the highest FIP quartiles in comparison to quartile 4 , the least fatty quartile $(1.4,3.83$, and 4.56 for quartiles 3 , 2, and 1 , 
Table 3. Results of logistical regression to predict pancreatic cancer based on the listed variables

\begin{tabular}{lcll}
\hline & OR & $p$ value & $95 \%$ CI \\
\hline P.S100 & 3.75 & 0.000171 & $1.9234408-7.993337$ \\
Age & 12.4648842 & 0.101058 & $0.9492523-1.003972$ \\
BMI & 0.9700735 & 0.327392 & $0.9123299-1.030905$ \\
Sex & 3.8942596 & 0.768181 & $0.4705407-1.781371$ \\
DM & 1.542316 & 0.251762 & $0.7490542-3.320319$ \\
\hline
\end{tabular}

The OR of each variable is corrected to the other listed variables. CI, confidence interval; OR, odds ratio; DM, diabetes mellitus type 2.

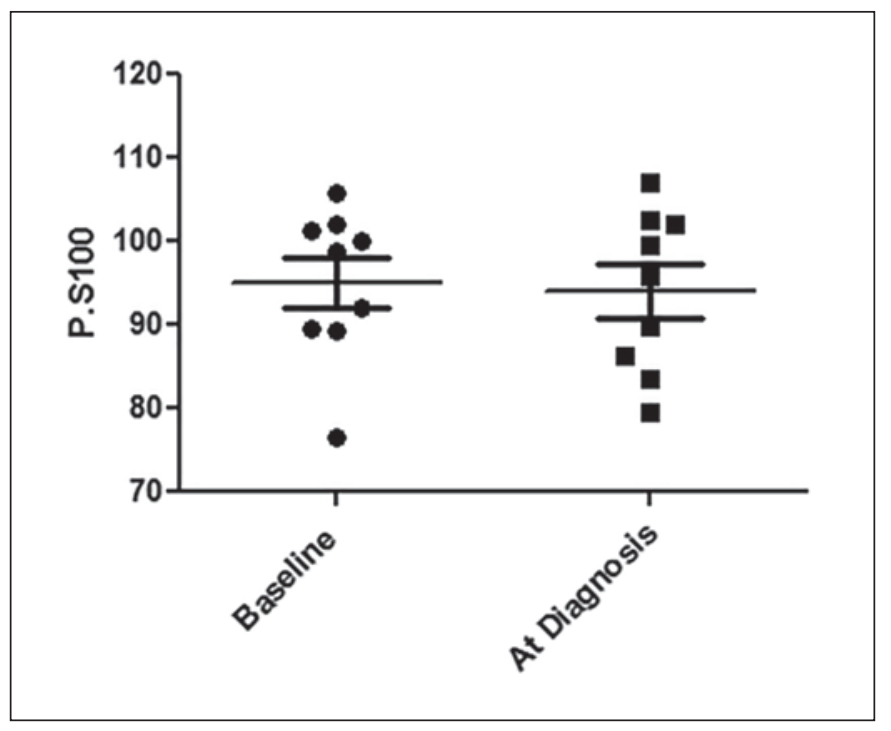

Fig. 2. FIP in 9 PDAC patients at baseline and at the time of diagnosis.

respectively; $p<0.001$ across all quartiles). The risk of pancreatic cancer increased significantly for the quartiles with a higher FIP than quartile 4 (quartile 2: 95\% CI 1.66-8.8 [ $p=0.00068]$ and quartile $1: 95 \%$ CI $2.0-10.37$ [ $p=0.00022]$ ).

To adjust for possible confounders, we performed a logistic regression. The risk of pancreatic cancer according to the FIP increased to 3.75 (95\% CI 1.92344087.993337; $p=0.000171$ ) after correcting for age, sex, BMI, and DM (Table 3 ).

We excluded the tumor-adjacent pancreas tissue when calculating FIP in our study. The increased intrapancreatic fat in the PDAC cases could be a process that is primary or secondary to tumor-associated inflammation. In order to examine the effect of PDAC on FIP, we compared the FIP of PDAC patients to their baseline FIP if they had $\mathrm{CT}$ results from $>3$ years prior to their PDAC diagnosis. We identified 9 cases with prior non-contrast CT results in our system when no pancreatic tumor was identifiable at baseline. There was no difference in the FIP before and after the diagnosis of PDAC in these cases (baseline P.S100 $95.02 \pm 3.035$ vs. $94.01 \pm 3.211 ; p=0.82$ ) (Fig. 2).

\section{Discussion}

The incidence of pancreatic cancer is increasing. The majority of patients have unresectable disease at the time of diagnosis, and earlier diagnosis could improve the 5 -year survival rate $[32,33]$. Identifying the patients who are at a risk of developing PDAC could potentially lead to early detection and potentially decreased mortality from this deadly disease. In this case-control study, we found that patients with pancreatic cancer had a lower P.S100 (i.e., a higher FIP) than controls. The risk ratio of pancreatic cancer increased significantly as we moved towards quartiles that represented higher FIP and a fattier pancreas. The higher FIP detected in the pancreatic cancer cases was independent of any possible effect of BMI, as cases had an overall lower BMI than controls. Upon adjustment for confounding variables including BMI, fatty pancreas was associated with an OR of 3.75 for PDAC.

Features of MetS (e.g., BMI and DM) as well as chronic alcohol consumption could be associated with fatty pancreas (Fig. 3). In our study, there were no significant differences in the alcohol history between cases and controls. Furthermore, adjustment for features of MetS made the association of FIP with PDAC even stronger in our series. Fatty pancreas based on histopathological analysis has been found to be associated with PDAC [28]. Similar to our findings, other authors have found that the association of fatty pancreas and PDAC remains significant even after adjusting for BMI and DM, thus proposing fatty pancreas as an independent risk factor for developing PDAC [28]. These results were confirmed by another study that reported greater fatty degeneration in the pancreatectomy specimens from PDAC cases than in controls who had undergone pancreatectomy for noncancerous lesions (72 vs. 44\%) [34]. Patients with hereditary pancreatitis secondary to germline PRSS1 mutations have been shown to have progressive intrapancreatic fatty infiltration that may put these patients at an increased risk of developing pancreatic cancer [35]. In support of fatty pancreas being a predisposing factor to pancreatic neoplastic transformation, Rebours et al. [36] found that the number of pancreatic precancerous (PanIN) lesions increased with the per- 
Fig. 3. Risk factors associated with FIP that lead to a possible increased risk of developing PDAC.

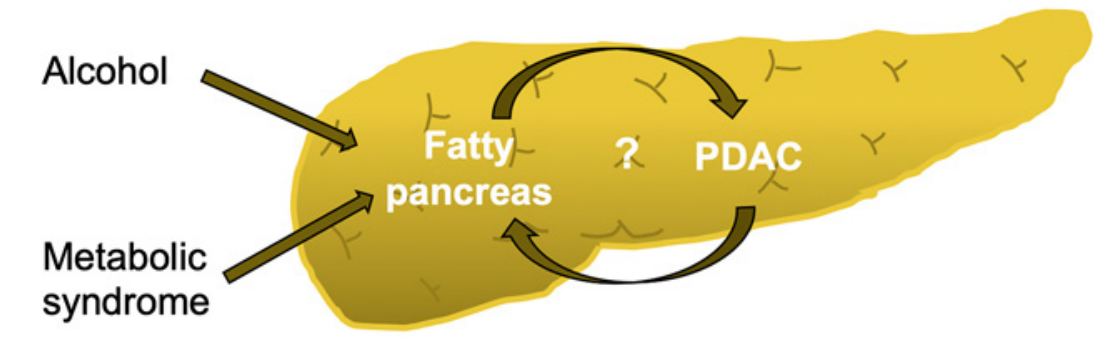

centage of intrapancreatic fatty infiltration, but that was not linked to the percentage of subcutaneous fat or BMI.

While initially perceived as a condition associated with MetS, pancreatic steatosis, and the subsequent fatty degeneration of the pancreas, can lead to a loss of $\beta$ cells which contributes to DM and worsening MetS. Similarly, fatty pancreas may be an independent risk factor for developing PDAC (stronger than other metabolic parameters) [29]. More recently, fatty pancreas, but not obesity and metabolic complications, was reported to be independently associated with the presence of intraductal papillary mucinous neoplasm, another precancerous pancreatic lesion [37]. Pancreatic steatosis may also impact the disease course by promoting the dissemination and lethality of pancreatic cancer; PDAC patients who underwent surgical resection and were found to be node-positive had significantly more fat cells in the pancreas than node-negative patients, associated with a worse survival [38].

We did not find a significant association between fatty pancreas and BMI or DM in our series. Some previous studies found a positive association of FIP with obesity and DM $[17,18,36,37,39-43]$. As there has been a global increase in obesity, it is possible that the prevalence of fatty pancreas is increasing in parallel. Our data point towards fatty pancreas being an independent increased risk factor for developing PDAC, thereby contributing to the burden of PDAC.

Mechanistically, fatty pancreas may increase PDAC risk via tissue remodeling and fibroinflammatory reactions $[15,16,28,36,44]$. Fatty pancreas, a subclinical condition, may cause a chronic inflammatory state in the pancreas, due to increased proinflammatory cytokines and adipokines, i.e., pathways known to increase malignant transformation [13,14, 19-21]. To avoid any resultant fat necrosis from the tumor itself, we selected areas for measuring pancreatic attenuation at a distance from the tumor site. However, based on the available clinical studies, it is still unclear if FIP results from the pancreatic cancer or precedes it. In the limited number of cases with CT images from before their PDAC diagnosis, we found no significant changes in IP before and after the PDAC diagnosis. This finding, if repeated in a larger sample, could argue in favor of pancreatic fat being a predisposing condition to cancer rather than a result of malignant transformation. This notion is supported by a recent study that reported on the inhomogeneous pancreas parenchyma as a predictive factor for pancreatic carcinogenesis, long before the diagnosis and independent of the tumor site [30].

While accurate assessment of fatty pancreas is only possible via histopathological examination of the tissue, the accumulating evidence suggests that imaging modalities can be used to accurately estimate pancreas fat $[20,28-31]$. Among the available imaging modalities, ultrasound may not be able to adequately visualize the pancreas, with fibrosis and fat both appear as hyperechogenic signals [31, 45]. MRI has been shown to give an accurate estimate of pancreatic steatosis, but it is costly and takes longer to perform [17, 41, 46-48]. CT is readily available, frequently performed, and relatively cheap. CT shows organ-related fat infiltration as a decrease in attenuation measured in Hounsfield units compared to the spleen [20]. Non-contrast study is preferred for diagnosing pancreatic steatosis as the normal pancreatic parenchyma in between the fatty areas can have contrast enhancement [46]. CT does, however, have limitations including undefined cut-off points for pancreatic steatosis, exposure to radiation, and the inability to diagnose mild degrees of focal fatty replacement. Nevertheless, several studies have shown the ability of CT to clinically evaluate pancreatic fat [29-31].

We are aware of the limitations of our study. It was carried out retrospectively, so we were not able to assess the duration and degree of exposure to alcohol and other factors possibly associated with the risk of developing pancreatic cancer $[5,12]$. We did not, however, observe a statistically significant difference in alcohol and tobacco use between cases and controls. We were also not able to correlate our CT findings with histopathological findings, but this is not possible anyway for the majority of PDAC patients due to their unresectability. A strength of our study was its design, which provided us with the opportunity to employ CT as a readily available tool for assessing fatty pancreas in all patients with PDAC at all stages, thus representing real-world practice. 


\section{Conclusion}

We found that fatty pancreas is associated with an increased risk of developing pancreatic cancer. Once confirmed in larger studies, these findings could help in identifying individuals who might be at a risk of developing pancreatic cancer.

\section{Acknowledgments}

The authors are grateful to Dr. Louis Fogg for his statistical assistance.

\section{Statement of Ethics}

This study was approved and conducted under the Rush University Medical Center institutional reviewboard (ORA\#15102401IRB01). Patient consent is waived for this retrospective study because the patient data were already collected and deidentified.

\section{Disclosure Statement}

There were no conflicts of interest.

\section{Funding Sources}

F.B. is supported by grants from the Rush Translational Sciences Consortium/Swim across America Organization, Brinson Foundation and Institute for Translational Medicine (ITM). This project was partially supported by the National Center for Advancing Translational Sciences of the National Institutes of Health (NIH) (grant No. 5UL1TR002389-02) that funds the ITM. The content is solely the responsibility of the authors and does not necessarily represent the official views of the NIH.

\section{Author Contributions}

Study concept and design (F.B.), acquisition of data (V.D., K.P., R.S., U.B., Y.M.C., and J.S.), analysis and interpretation of data (F.B.); drafting of the manuscript (V.D. and F.B.); critical revision of the manuscript for important intellectual content (F.B.); statistical analysis (F.B.).

\section{References}

1 Vincent A, Herman J, Schulick R, Hruban $\mathrm{RH}$, Goggins M. Pancreatic cancer. Lancet. 2011 Aug;378(9791):607-20.

2 Rahib L, Smith BD, Aizenberg R, Rosenzweig AB, Fleshman JM, Matrisian LM. Projecting cancer incidence and deaths to 2030: the unexpected burden of thyroid, liver, and pancreas cancers in the United States. Cancer Res. 2014 Jun;74(11):291321.

3 Yeo TP. Demographics, epidemiology, and inheritance of pancreatic ductal adenocarcinoma. Semin Oncol. 2015 Feb;42(1):8-18.

4 Farrell JJ. Intraductal papillary mucinous neoplasm to pancreas ductal adenocarcinoma sequence and pancreas cancer screening. Endosc Ultrasound. 2018 Sep-Oct;7(5):3148.

5 Hassan MM, Bondy ML, Wolff RA, Abbruzzese JL, Vauthey JN, Pisters PW, et al. Risk factors for pancreatic cancer: case-control study. Am J Gastroenterol. 2007 Dec;102(12): 2696-707.

6 Johnson CD, Schwall G, Flechtenmacher J, Trede M. Resection for adenocarcinoma of the body and tail of the pancreas. Br J Surg. 1993 Sep;80(9):1177-9.

7 Ozawa F, Friess H, Künzli B, Shrikhande SV, Otani T, Makuuchi M, et al. Treatment of pancreatic cancer: the role of surgery. Dig Dis. 2001;19(1):47-56.

8 Katz MH, Wang H, Fleming JB, Sun CC, Hwang RF, Wolff RA, et al. Long-term survival after multidisciplinary management of resected pancreatic adenocarcinoma. Ann Surg Oncol. 2009 Apr;16(4):836-47.

9 Hidalgo M. Pancreatic cancer. N Engl J Med. 2010 Apr;362(17):1605-17.

10 Siegel RL, Miller KD, Jemal A. Cancer Statistics, 2017. CA Cancer J Clin. 2017 Jan;67(1): 7-30.
11 Arslan AA, Helzlsouer KJ, Kooperberg C, Shu XO, Steplowski E, Bueno-de-Mesquita HB, et al.; Pancreatic Cancer Cohort Consortium (PanScan). Anthropometric measures, body mass index, and pancreatic cancer: a pooled analysis from the Pancreatic Cancer Cohort Consortium (PanScan). Arch Intern Med. 2010 May;170(9):791-802.

12 Rosato V, Tavani A, Bosetti C, Pelucchi C, Talamini R, Polesel J, et al. Metabolic syndrome and pancreatic cancer risk: a case-control study in Italy and meta-analysis. Metabolism. 2011 Oct;60(10):1372-8.

$13 \mathrm{Ou} \mathrm{HY}$, Wang CY, Yang YC, Chen MF, Chang CJ. The association between nonalcoholic fatty pancreas disease and diabetes. PLoS One. 2013 May;8(5):e62561.

14 Assimacopoulos-Jeannet F. Fat storage in pancreas and in insulin-sensitive tissues in pathogenesis of type 2 diabetes. Int J Obes Relat Metab Disord. 2004 Dec;28(S4 Suppl 4):S53-7.

$15 \mathrm{Wu}$ Q, Chen G, Wu WM, Zhou L, You L, Zhang TP, et al. Metabolic syndrome components and risk factors for pancreatic adenocarcinoma: a case-control study in China. Digestion. 2012;86(4):294-301.

16 Abbruzzese JL, Andersen DK, Borrebaeck CA, Chari ST, Costello E, Cruz-Monserrate Z, et al. The Interface of Pancreatic Cancer with Diabetes, Obesity, and Inflammation: Research Gaps and Opportunities: Summary of a National Institute of Diabetes and Digestive and Kidney Diseases Workshop. Pancreas. 2018 May/Jun;47(5):516-25.

17 Wong VW, Wong GL, Yeung DK, Abrigo JM, Kong AP, Chan RS, et al. Fatty pancreas, insulin resistance, and $\beta$-cell function: a population study using fat-water magnetic resonance imaging. Am J Gastroenterol. 2014 Apr;109(4):589-97.
18 Catanzaro R, Cuffari B, Italia A, Marotta F. Exploring the metabolic syndrome: nonalcoholic fatty pancreas disease. World J Gastroenterol. 2016 Sep;22(34):7660-75.

19 Maggio AB, Mueller P, Wacker J, Viallon M, Belli DC, Beghetti M, et al. Increased pancreatic fat fraction is present in obese adolescents with metabolic syndrome. J Pediatr Gastroenterol Nutr. 2012 Jun;54(6):720-6.

20 Hori M, Onaya H, Hiraoka N, Yamaji T, Kobayashi H, Takahashi M, et al. Evaluation of the degree of pancreatic fatty infiltration by area-based assessment of CT images: comparison with histopathology-based and CT attenuation index-based assessments. Jpn J Radiol. 2016 Oct;34(10):667-76.

21 Tariq H, Nayudu S, Akella S, Glandt M, Chilimuri S. Non-Alcoholic Fatty Pancreatic Disease: A Review of Literature. Gastroenterol Res. 2016 Dec;9(6):87-91.

22 Khandekar MJ, Cohen P, Spiegelman BM. Molecular mechanisms of cancer development in obesity. Nat Rev Cancer. 2011 Nov; 11(12):886-95.

23 Smits MM, van Geenen EJ. The clinical significance of pancreatic steatosis. Nat Rev Gastroenterol Hepatol. 2011 Mar;8(3):169-77.

24 Berger NA. Obesity and cancer pathogenesis. Ann NY Acad Sci. 2014 Apr;1311(1):57-76.

25 Hori M, Kitahashi T, Imai T, Ishigamori R, Takasu S, Mutoh M, et al. Enhancement of carcinogenesis and fatty infiltration in the pancreas in N-nitrosobis(2-oxopropyl)amine-treated hamsters by high-fat diet. Pancreas. 2011 Nov; 40(8):1234-40.

26 Philip B, Roland CL, Daniluk J, Liu Y, Chatterjee D, Gomez SB, et al. A high-fat diet activates oncogenic Kras and COX2 to induce development of pancreatic ductal adenocarcinoma in mice. Gastroenterology. 2013 Dec; 145(6):1449-58. 
27 Chang HH, Moro A, Takakura K, Su HY, Mo A, Nakanishi M, et al. Incidence of pancreatic cancer is dramatically increased by a high fat, high calorie diet in KrasG12D mice. PLoS One. 2017 Sep;12(9):e0184455.

28 Hori M, Takahashi M, Hiraoka N, Yamaji T, Mutoh M, Ishigamori R, et al. Association of pancreatic Fatty infiltration with pancreatic ductal adenocarcinoma. Clin Transl Gastroenterol. 2014 Mar;5(3):e53.

29 Fukuda Y, Yamada D, Eguchi H, Hata T, Iwagami Y, Noda T, et al. CT Density in the Pancreas is a Promising Imaging Predictor for Pancreatic Ductal Adenocarcinoma. Ann Surg Oncol. 2017 Sep;24(9):2762-9.

30 Gonoi W, Hayashi TY, Okuma H, Akahane M, Nakai Y, Mizuno S, et al. Development of pancreatic cancer is predictable well in advance using contrast-enhanced CT: a case-cohort study. Eur Radiol. 2017 Dec;27(12):4941-50.

31 Kim SY, Kim H, Cho JY, Lim S, Cha K, Lee $\mathrm{KH}$, et al. Quantitative assessment of pancreatic fat by using unenhanced CT: pathologic correlation and clinical implications. Radiology. 2014 Apr;271(1):104-12.

32 Bilimoria KY, Bentrem DJ, Ko CY, Ritchey J, Stewart AK, Winchester DP, et al. Validation of the 6th edition AJCC Pancreatic Cancer Staging System: report from the National Cancer Database. Cancer. 2007 Aug;110(4): 738-44.

33 Siegel R, Ma J, Zou Z, Jemal A. Cancer statistics, 2014. CA Cancer J Clin. 2014 Jan-Feb; 64(1):9-29.
34 Tomita Y, Azuma K, Nonaka Y, Kamada Y, Tomoeda M, Kishida M, et al. Pancreatic fatty degeneration and fibrosis as predisposing factors for the development of pancreatic ductal adenocarcinoma. Pancreas. 2014 Oct;43(7): 1032-41.

35 Singhi AD, Pai RK, Kant JA, Bartholow TL, Zeh HJ, Lee KK, et al. The histopathology of PRSS1 hereditary pancreatitis. Am J Surg Pathol. 2014 Mar;38(3):346-53.

36 Rebours V, Gaujoux S, d'Assignies G, Sauvanet $A$, Ruszniewski $P$, Lévy $P$, et al. Obesity and Fatty Pancreatic Infiltration Are Risk Factors for Pancreatic Precancerous Lesions (PanIN). Clin Cancer Res. 2015 Aug;21(15): 3522-8.

37 Kashiwagi K, Seino T, Fukuhara S, Minami K, Horibe M, Iwasaki E, et al. Pancreatic Fat Content Detected by Computed Tomography and Its Significant Relationship with Intraductal Papillary Mucinous Neoplasm. Pancreas. 2018 Oct;47(9):1087-92.

38 Mathur A, Zyromski NJ, Pitt HA, Al-Azzawi $\mathrm{H}$, Walker JJ, Saxena R, et al. Pancreatic steatosis promotes dissemination and lethality of pancreatic cancer. J Am Coll Surg. 2009 May;208(5):989-94.

39 Walters MN. Adipose atrophy of the exocrine pancreas. J Pathol Bacteriol. 1966 Oct;92(2): 547-57.

40 Toyama N, Kamiyama H, Suminaga Y, Namai K, Ota M, Konishi F. Pancreas head carcinoma with total fat replacement of the dorsal exocrine pancreas. J Gastroenterol. 2004 Jan;39(1):76-80.

41 Lee JS, Kim SH, Jun DW, Han JH, Jang EC, Park JY, et al. Clinical implications of fatty pancreas: correlations between fatty pancreas and metabolic syndrome. World J Gastroenterol. 2009 Apr;15(15):1869-75.
42 Rosso E, Casnedi S, Pessaux P, Oussoultzoglou E, Panaro F, Mahfud M, et al. The role of "fatty pancreas" and of BMI in the occurrence of pancreatic fistula after pancreaticoduodenectomy. J Gastrointest Surg. 2009 Oct; 13(10):1845-51.

43 Makay O, Kazimi M, Aydin U, Nart D, Yilmaz F, Zeytunlu M, et al. Fat replacement of the malignant pancreatic tissue after neoadjuvant therapy. Int J Clin Oncol. 2010 Feb;15(1):8892.

44 Takahashi M, Hori M, Ishigamori R, Mutoh M, Imai T, Nakagama H. Fatty pancreas: A possible risk factor for pancreatic cancer in animals and humans. Cancer Sci. 2018 Oct; 109(10):3013-23.

45 Ricci C, Longo R, Gioulis E, Bosco M, Pollesello P, Masutti F, et al. Noninvasive in vivo quantitative assessment of fat content in human liver. J Hepatol. 1997 Jul;27(1): 108-13.

46 Kim HJ, Byun JH, Park SH, Shin YM, Kim $\mathrm{PN}, \mathrm{Ha} \mathrm{HK}$, et al. Focal fatty replacement of the pancreas: usefulness of chemical shift MRI. AJR Am J Roentgenol. 2007 Feb;188(2): 429-32.

47 Lingvay I, Esser V, Legendre JL, Price AL, Wertz KM, Adams-Huet B, et al. Noninvasive quantification of pancreatic fat in humans. J Clin Endocrinol Metab. 2009 Oct;94(10): 4070-6.

48 Pezzilli R, Calculli L. Pancreatic steatosis: is it related to either obesity or diabetes mellitus? World J Diabetes. 2014 Aug;5(4):415-9. 